\title{
Evaluating a Visual Search Interface
}

\author{
Maria Henkel ${ }^{\mathrm{a}}$ and Maurice Schleußinger $\mathrm{ab}^{*}$ \\ ${ }^{a}$ Department of Information Science, Heinrich Heine University Düsseldorf, \\ ${ }^{b}$ Center of Information and Media Technology, Heinrich Heine University \\ Düsseldorf,
}

Universitätsstraße 1, D-40225 Düsseldorf, Germany

*Corresponding Author: maurice.schleussinger@hhu.de

\begin{abstract}
Evaluation of information visualization and especially visual information systems is challenging. Metrics and methods need to be chosen with the intend of gaining specific and relevant evaluation data to prove the value of such systems. We suggest an evaluation based on the Information Service Evaluation Model (ISE) for information systems with visual result representation. We present the results of a case study on the testing and implementation of a visual search engine in business.
\end{abstract}

Keyword: Development of Information and Knowledge Services, Information Visualization, Information Retrieval

\section{Introduction}

Result representation of common web-based search engines like Google, Baidu and Yandex (Alexa Internet Inc., 2017) has not changed much over the years. Since their beginning in 1990 (Stock \& Stock, 2013), Result representation has been a list of text snippets foreach search result. There is no connection between similar search results and results on the second, third and following pages are barely noticed. A visual search engine, combining the same aspects of information retrieval with information visualization, could show us the "big picture" of our search results. Information visualization, a research focus of growing interest over the recent years, is "an increasingly important part of cognitive systems. Visual displays provide the highest bandwidth channel from the computer to the human", which helps us to understand "large-scale and small-scale features of the data" observed and supports us in gaining new insight, for example, by recognizing properties of and also problems with the data, that were not anticipated before (Ware, 2004). As a great quantity of information can be processed more quickly with the help of visualization, it is not only helpful for " scientists and analysts", but also for "commercial and personal use" (Heer, Van Ham, Carpendale, Weaver, \& Isenberg, 2008). Especially businesses recognize "the need for more effective tools for extracting knowledge from the data warehouses they are gathering" (Heer et al., 2008) and are starting to implement information visualization 
in their work flows. We are convinced that visualization has value for interactive information retrieval and information discovery. To prove this value, however, visual information systems have to be evaluated and compared to conservative information systems. Unfortunately, while designing information visualization systems is already a complex task (Ware, 2004), their evaluation might prove to be even more challenging (Fekete, Wijk, Stasko, \& North, 2008):

"So why is identifying the value of InfoVis so difficult? To help answer that question, let us turn to what is probably the most accepted definition of InfoVis, one that comes from Card, Mackinlay, and Shneiderman and that actually is their definition for "visualization." They describe visualization as "the use of computer-supported, interactive visual representations of data to amplify cognition'. (Card, Mackinlay, \& Shneiderman, 1999) The last three words of their definition communicate the ultimate purpose of visualization, to amplify cognition. So, returning to our discussion above, is the amplification of cognition something with a ground truth that is easily and precisely measurable? Clearly it is not and so results the key challenge in communicating the value of InfoVis."

Indeed, even simple systems contain a multitude of parameters - some easily overlooked - and the smallest changes can, again, change many parameters at once. Empirically speaking, it is nearly impossible to detect all dependent variables, which explain a measured change. Furthermore, the choice of independent variables, that is adequate measurements, is also a topic of great controversy (Ellis \& Dix, 2006).

We see many of these challenges properly addressed by (Ellis \& Dix, 2006) and share their wish for precise, well-planed and result-oriented evaluation methods. By reviewing existing evaluation approaches for information visualization systems with and without search functionality, and by adapting them for a case study with a working system from the industry, we hope to contribute to previous research and future projects in science and industry.

For this case study, we adapt the Information Service Evaluation model (ISE) (Schumann \& Stock, 2014) for information systems and apply the derived evaluation methods for Knowde, an interactive information retrieval system with visual result representation (Schleußinger \& Henkel, 2018). In the following, we will discuss earlier attempts at developing and evaluating visual information systems and related work. We will then introduce the ISE model (Schumann \& Stock, 2014) and Knowde, the visual 
search engine evaluated in the case study. After describing our interview methods and questionnaire, results will be presented and discussed.

\section{Related Work}

For classic information retrieval systems, the ranking of search results is very important as it is a direct indicator of the success of a search query. Information retrieval system designers ask themselves: Can I give a user the most relevant result for his search terms? For this, measurements like recall and precision or mean average precision are calculated with annotated test collections. A retrieval system with focus on the result presentation (visualization) also wishes for adequate search result ranking, but seldom focuses on this aspect itself as the main topic of research. Instead, the evaluation of the visualization method becomes much more important. The key question is: Does my visualization convey the intended information? And how can I measure this?

The idea of combining search and visualization in one system is not new. Earlier approaches, the information space prototype by (Rohrer \& Swing, 1997), SENTINEL and NIRVE (Cugini, Laskowski, \& Sebrechts, 2000), attempted 3D visualizations, requiring the user to navigate a 3-dimensional space, using mouse and keyboard input and a 2-dimensional computer monitor. User evaluation yielded mixed results.

Yi, ah Kang, Stasko, and Jacko, (2007) developed what they called Jigsaw, a system which mainly focused on relationships between reports without any search query functionality. They mention the need for evaluation without actually conducting any. In a later article, however, they introduce a throughout evaluation (Bederson, Shneiderman, \& Wattenberg, 2002) with 16 graduate students measuring task performance, number of queries and completion time. Additionally, their setup encouraged the test subjects to take notes, which were also collected and analyzed.

Grierson, Corney, and Hatcher, (2015) tested “. . . whether a 2.5D graphical representation of a multimedia data landscape produces quantifiable improvements in a user's ability to assess its contents" by letting 16 students do the same tasks with their system and an established and more general software (Microsoft File Manager). They report an improvement in accuracy and speed but also some users getting lost while using their system. They measure time, accuracy and collect observation notes as well as thinking aloud exercises and finally, a questionnaire.

Heer and Boyd, (2005) developed an interactive User Interface called Vizster for the browsing of social networks. Its visualization is realized by a graph which centers 
around the user and displays their friends and their connectedness (via nodes and lines) as well as possible clusters of friends. It also features a list of details for a selected user and allows a simple keyword search. They conclude, that future research is to be conducted on the extend of applicability of their technique to "more traditional analytical domains" in junction with a more "rigorous evaluation [...] to evaluate benefits and tradeoffs."

FacetMap (Smith et al., 2006) features a search and filter system which puts emphasis on the navigation of hierarchical categories like date and type. WebSearchViz (Nguyen \& Zhang, 2006) even complements existing Google search results with a browser plugin that visualizes document closeness to a given query.

The interactive visual information retrieval system of Klouche, Ruotsalo, Micallef, Andolina, and Jacucci (2017) features a within-subject laboratory study as evaluation. They distinguish between two search tasks and define a baseline system (traditional information retrieval system) for comparability. Furthermore, they vary the order of system and tasks for their test subjects.

$\mathrm{Xu}$, Jin, and Lau (2009) executed a user study with 30 graduate students. They used Google as benchmark and formulated 30 questions, designed to require an explorative web search to adequately complete them. Each user had to answer 15 of these questions randomly assigned to them. Measurements included completion time and user satisfaction on a 0 to 10 scale. The researchers intellectually assessed the quality of the answers given and compared the mean of these scores both for the Google search and their visual system.

Overall we see evaluations of visualization systems with or without a search component varying in extent and quality. Ellis and Dix (2006) reviewed " 65 papers describing new visualization application or techniques" and found that only 12 of them described any evaluation and only 2 were deemed "successful" by the authors. They list several challenges and difficulties in visualization evaluation:

\subsection{Complexity}

Every design detail potentially affects the usability and therefore the evaluation results. This includes color choices, button layout and so on. There are multiple stages at which user interaction takes place and success might be wrongly dedicated to a change in, for example, the overall visualization technique, while it might be caused by a trivial change of font size or color. 


\subsection{Diversity}

Ellis and Dix (2006) report a lack of good real data sets and that often, artificial data is used instead. The choice in tasks might influence the outcome of the evaluation since certain tasks are more suited for certain visualization types. Plus, "Visualisations are often at their best for more exploratory tasks, but these are precisely the tasks that are hardest to replicate in an experiment" (Ellis \& Dix, 2006). Therefore, users should be encouraged to get accustomed to a new system before completing tasks. Ellis and Dix (2006) criticize how researchers tend to use students as convenient test subjects. Especially for realistic tasks in specific domains "participants need a clear understanding of the problem [...] and [...] the data itself" (Ellis \& Dix, 2006).

\subsection{Measurement}

To enable applying numerical measurement one needs a valid comparison. It should be avoided to compare old, outdated software which was designed for a different purpose to a newly specially designed system. Almost any non-trivial existing solution would vary in many aspects so it should be difficult to assign a measured effect to a certain change in functionality, design, performance, etc. Ellis and Dix (2006) put it bluntly: "[Y] ou cannot evaluate a visualisation... or at least any evaluation cannot tell you, in itself, that the visualisation works or doesn't work." and any attempted explanation in regard to the reason of a measured effect cannot be perfect "because our base knowledge of human perception and cognition is incomplete".

Before one now stops to work on any evaluation even remotely related to visualization, they provide a general guideline to follow: "[I]f empirical evaluation complements reasoned justification then it can lead to a reliable and strong validation of the visualization". The justification can therefore be conducted from existing published results of experiments, analysis, and empirical data as well as expert opinion and common sense. Evaluation could feature empirical evaluation, user studies, timing data, etc. but also peer reviews and a comparison with previous work (Ellis \& Dix, 2006). They continue to suggest a more explorative evaluation approach as an alternative to summative assessment (direct comparison of two systems) and formative ones (improving design). "[...] the purpose of explorative evaluation is to find out, to provide knowledge"(Ellis \& Dix, 2006).

Ware (2004) created a very extensive work on the many aspects of designing and analyzing visual search interfaces and also provides a guideline for conduction experiments for Information Visualization interfaces. It starts by defining the magnitude of possible discoveries made from research on the subject. Besides 
discovering the "uncover fundamental truths and test theories" and discovering "the nature of the world" (Ware, 2004, p. 393), one can also hope to "make an objective comparison between two or more display methods" or systems as well as "measure task performance" and even "ascertain user preferences for different display methods" (p. 394). This research covers the latter. While exploring different approaches for research on this topic, Ware (2004) found the structural analysis to be appropriate for studying computer interfaces. Methods include task analysis, test bench applications, semistructured interviews and rating scales.

In this spirit of Ware (2004) and Ellis and Dix (2006) we suggest the ISE Model by Schumann and Stock (2014). With its "holistic and comprehensive approach" the model qualifies as a general guideline for evaluating an information service. While it was not explicitly designed for visual information services, it can still be adapted for such systems. Ware (2004) provides guidelines for evaluation techniques to use for visual systems and mentions many of the methods proposed by ISE as well. This includes but is not limited to structured or semi-structured interviews, Likert (rating) scales and task testing. The ISE Model is explained in more detail in section 4, where it is used for our evaluation. 

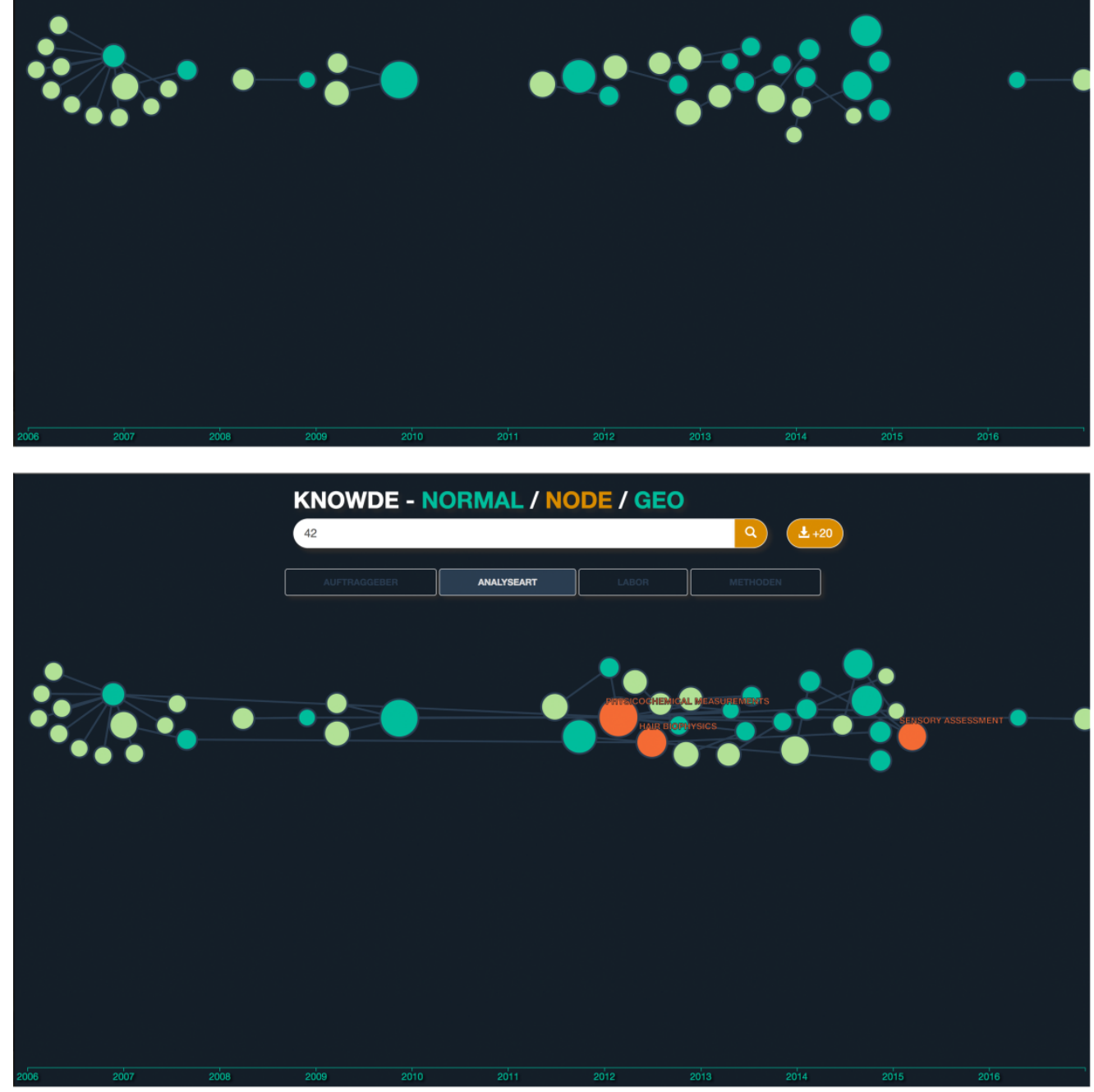

\section{KNOWDE - NORMAL / NODE / GEO}

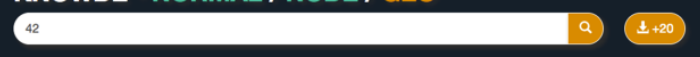

AMaLrSEART
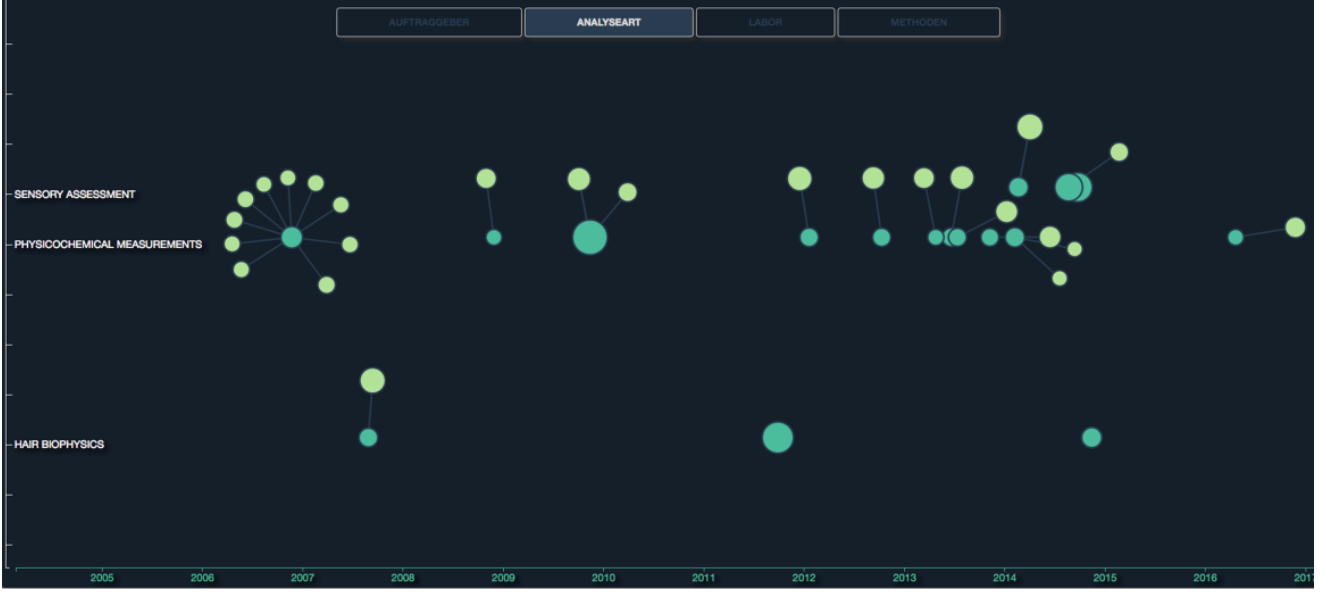

Figure 1:Screenshot of the Knowde system with its three 329 modes (top to bottom) 


\section{Description of the KNOWDE System}

Knowde (Knowledge Node), is an information retrieval system that employs elements of information visualization for result presentation. It adapts network graphs into a web search interface, using them as the sole means of search result representation and main focus for user interaction. The visual system features three different modes as shown in Figure 1. They consist of a fixed search bar at the top and, depending on the mode, buttons for category selection. The entirety of the screen is dominated by the actual visualization of search results as network graphs. Resulting from the specific structure of the company's data set there are two connected node types: documents (blue) and their attachments (green). The search function features a powerful search index which enables access to all textual information provided in the many file formats of the provided data set. Result relevance is expressed in the size of the nodes. Only small chunks (around 20 documents with their attachments) of the full result set are displayed initially, to reduce visual clutter, but more results can be loaded on demand. The user can fluidly zoom and drag in the visualization at any point using the computer's mouse. All resulting reports are sorted on a time axis based on their creation date. Up to this point, we describe mode 1 , which is the basis for all other modes.

In mode 2, categorical information is displayed in the form of a third type of nodes (orange). These additional nodes are sized by absolute occurrences in the result set and are connected to all report nodes with the corresponding category.

In mode 3, the same categorical information is displayed on the y-Axis of the window. The white stripes on the axis represent the total amount of values for the selected category, but only matching values are labeled. The value labels are sorted alphabetically and scale on zooming and panning together with the nodes. Therefore, at any point the category information for visible nodes can be retrieved and never changes its relative position.

As Knowde was developed in cooperation with a large, internationally operating company, a real data set was used and every step took place in a real work environment, tackling real challenges in information retrieval and visualization for organizations with a huge amount of heterogeneous data.

Schleußinger and Henkel (2018) give more details on the data set as well as the design process and the technical implementation of Knowde.

\section{Methods (Evaluation design \& analysis)}

To again summarize Ellis and Dix (2006): A good evaluation for visualization systems should be based on real data, with relevant test subjects. It should not be limited to improve a system in small steps, but instead focus heavily on the exploration of truly 
novel insight.

Evaluation of the Knowde prototype is realized by conducting interviews with 24 employees of a mayor international cooperation, aided by a digital questionnaire. The data set used for the prototype contains real company data which is regularly generated and used by the employees. The interview consists of an introduction, where the system is explained, followed by the questionnaire regarding information user, the objective information service quality, perceived information system quality and information acceptance, according to the dimensions of ISE. Time is excluded, as no long-term analysis was possible. The interview is completed with a free testing phase, where each user can test all modes of the prototype as they want.

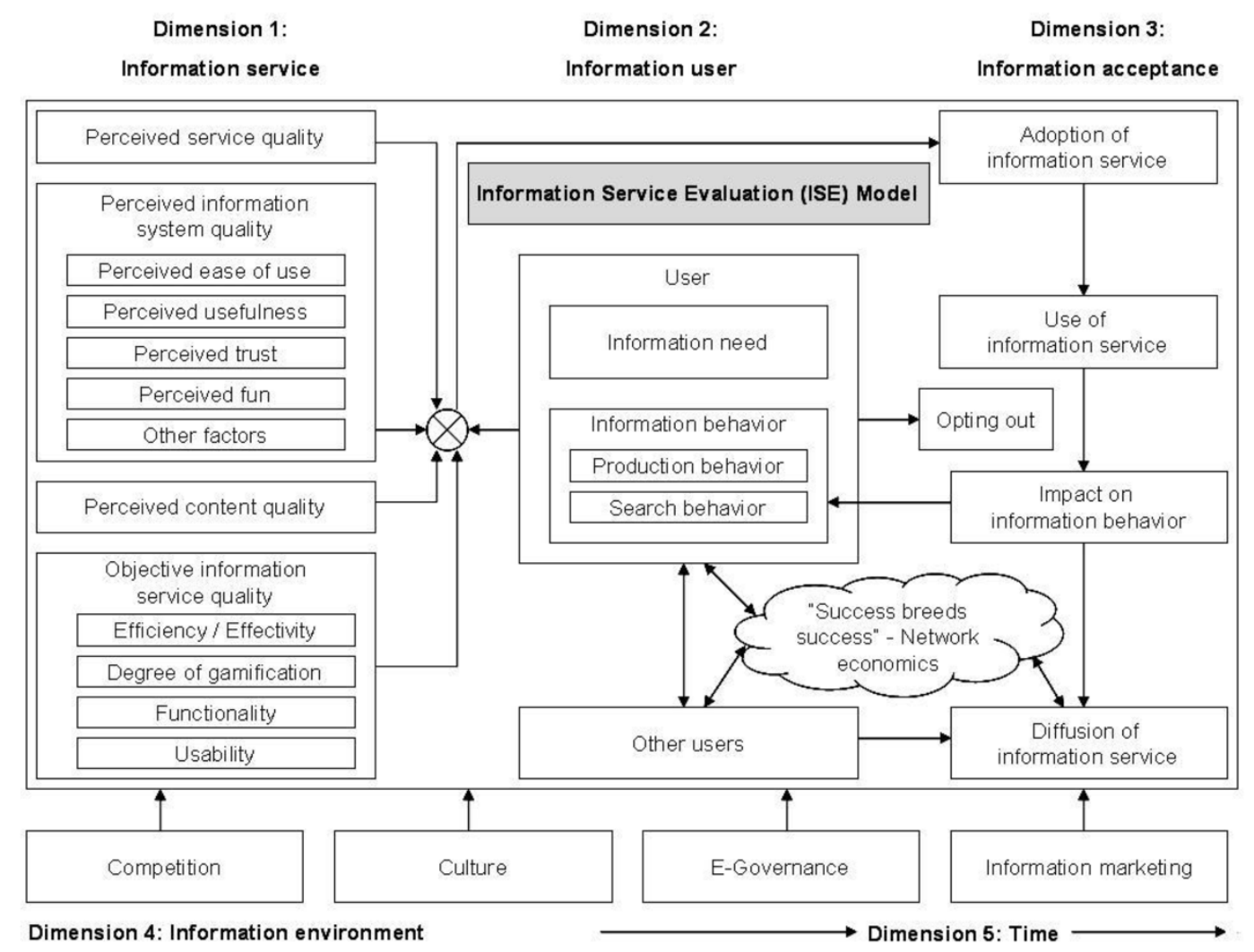

Figure 2: The Information Service Evaluation Model (Schumann \& Stock, 2014)

\subsection{ISE as structural guideline}

The ISE model contains five dimensions and allows extensive evaluation of key areas of any information service. Figure 2 gives an overview of the five dimensions which measure the quality and success of such a system. Schumann and Stock (2014) also provide a number of evaluation techniques and methods for analyzing these dimensions.

Information Service Quality. The first dimension, the Information Service Quality, is further sub-categorized: First, for the perceived service quality, one has to evaluate how the service as a whole is perceived by test subjects. As part of the critical incident 
technique and as a thinking-aloud task, test subjects were asked to comment on anything extraordinary or unusual (be it in a positive or negative fashion) or basically mention things which came into their minds during the interviews. The qualitative feedback in form of these comments was transcribed, sorted thematically and summarized in terms of key aspects.

Secondly the perceived system quality is analyzed in some detail for each mode of the system in regard to the ease of use, the usefulness, and fun. Perceived trust was questioned for the overall system since there are no differences in the way the three modes work in that regard (data handling etc.). Users were asked to rate statements on a 7-point scale (Likert, 1932) ranging from not at all to totally. As subjects "must be familiar with the system in order to make correct assessments" (Schumann \& Stock, 2014) these questions were asked after throughout interaction with each mode.

The objective information service quality should play a major role in visualization system assessment and is being evaluated by measuring and testing efficiency (doing things right), effectiveness (doing the right things), functionality (doing all things it should) and usability (is the system user-friendly). Efficiency can be tested by measuring the system's performance during use and the speed in which queries are processed and results are displayed. An analysis of effectiveness would test the retrieval capabilities of the system.

Since the basic retrieval system was adapted from existing solutions no further analysis of the effectiveness takes place. As previously explained, the effectivity of the retrieval component can be tested with various calculations and collections. But the evaluated system adapts existing information retrieval functionality as provided by Elasticsearch. While more extensive evaluation could feature such tests to achieve maximum completeness in regard to the ISE framework, it will not be approached in this research. On the other hand, the usability should be of the greatest interest independent of evaluation scope. Visualization success stands and falls with the success of user interaction and understanding of the elements displayed. Hence, the usability is tested by following guidelines by Nielsen (1993) for task-based testing. Users are given retrieval tasks designed to test their understanding of the interface elements and result representation as well as relationships between documents. Overall, ten questions are to be solved by 24 test subjects. The questions vary in difficulty (in rising order throughout the questionnaire) and aim to test the understanding of all key functions of the system. In combination with the free testing they also provide the means of getting the user accustomed to the system.

Information User. The analysis of the person who is expected to use an information system is crucial since any information system is created to satisfy a person's 
information need, the wish to gain new insight and knowledge. One has to assess whether the user is an information professional, how much knowledge about the service the user already has, as well as their information need and behavior. Furthermore, some "general person-related factors such as gender, age, digital native/immigrant and culture" should be gathered (Schumann \& Stock, 2014) .

For us, characterization of the Information User includes a simple questionnaire at the beginning of the interview. Questions regard job, gender, age, whether the person has any skills in information processing, uses search engines regularly, the level of computer/smartphone use and familiarity with the given data. This should allow to infer what kind of user took the questionnaire (Information professional, Professional end user or End user (information layman)) and in summary how representative the evaluation can be.

Information Acceptance. How well is the system accepted by the target user group? There is a difference between using a system and adopting it, where the latter does not imply the former because a user can stop using an adapted system after a while. Only if an information need can be satisfied with the introduced system, system adopting takes place (Schumann \& Stock, 2014). Questionnaires about the adoption, usage, impact and diffusion, also in relation to other users, are to take place. The topic is only lightly touched in this case study. It requires a long-term evaluation of an actually deployed system and repeated interviews. Continued cooperation with a company could prove exceptionally valuable, given the possibility to record and influence information acceptance for a real use case. The questionnaire features two questions on information acceptance:

- Would you want to continue using this system in your work?

- Should the company implement this system? (or parts of it?)

This question to the test subjects collects their spontaneous reaction after one time usage of the system and means to assess if adoption is at all possible.

Information Environment. What is the context of the information system and its (potential) users? Multiple cultural and economic factors are to be considered. Insight can be gained by consulting relevant research, company reports or interviews with the stakeholders themselves(Schumann \& Stock, 2014).

\subsection{Interview parameters}

Between- vs. Within-Subject Testing. Additionally, there is the problem of betweensubject testing vs. within-subject testing to address. The former would require each mode to be tested by a different user to avoid the subjects getting accustomed to the 
system. The latter would mean testing all systems with each user. As explained, the first mode provides the baseline of comparison between the other two. Therefore, users would get accustomed to some degree and can apply their new gained knowledge to the novel two modes. To somewhat randomize the effect of getting again better in using the system after seeing a second mode, subjects are randomly assigned to two groups who will have a different order of testing. The first group tests mode 1, then mode 2 and finally mode 3 while the other group tests mode 1, mode 3 and lastly mode 2 .

Experimenter. Since the experimenter is also the system designer, he would not help any test subject at all, except for the opening explanation and obvious input errors like for example not using the mouse wheel to zoom, or how to click on an element to activate it. Experimenters bias should therefore be kept to a minimum while the requirement of having an experimenter with "extensive knowledge of the application and its user interface" (Nielsen, 1993) is kept as well.

Formal Parameters. The formal parameters of the interview are derived from the guidelines provided by (Nielsen, 1993) and are as follows:

The subjects have not used the system before, as required for task-based testing.

Users are invited to a typical work environment, which is an office room where a seat is placed in front of a computer. All sound sources as for example cellphones and computer notifications are disabled. The questionnaire is opened in a browser window as well as the search interface (while not visible at the beginning of the interview). Prior to any questions, the user is given the following introduction:

1. The system is experimental and there is no guarantee that it will be introduced into a productive context at the company.

2. The system is being tested, not the user. Hence, users need not be afraid of making mistakes.

3. The system is new and untested and errors or other problems can occur.

4. The test subject can stop at any time for any or no reason.

5. There will be logs of mouse clicks, search queries and the time between interactions.

6. All data is collected anonymously and kept confidential.

7. There is no audio or video recording, only the questionnaire, interviewers notes and logs.

8. Comments of any kind are welcome. The test subject is encouraged to mention any positive, negative or unexpected incidents.

9. Questions will be answered as far as they do not compromise the task tests.

At this point, the first part of the questionnaire is answered by the user. 


\subsection{Statistical Analysis}

Results of the quantitative results of the interviews are shown in form of tables and plots as appropriate. The aggregations are described as they are used. Furthermore, statistical analysis will follow. At this time, it should be noted that there is an ongoing discussion in the scientific community in regard to the use of parametric statistics for ordinal data such as Likert scales. Some argue "mean (and standard deviation) are inappropriate for ordinal data" (Jamieson, 2004), the median should be used exclusively instead and using parametric test for ordinal data is one of "the seven deadly sins of statistical analysis" (Kuzon, Urbanchek, \& Mccabe, 1997). Others state "Parametric statistics can be used with Likert data, with small sample sizes, with unequal variances, and with non-normal distributions" (Norman, 2010). So, for further analysis the median is calculated for the non-parametric results, some data is summed up and the MannWhitney-Wilcoxon test or Mann-Whitney $U$ test is used for hypothesis testing of the Likert scales and the ranking of the different modes.

The Mann-Whitney U test is even applicable for data which does not have a normal distribution, like possibly skewed Likert results. Furthermore, we apply it for nonparametric (ordinal) data which is not paired (subjects were not asked twice). The test provides a $p$-value of significance for a given null hypothesis. For this thesis the current $\mathrm{R}$ implementation is used which bases its calculations on the work of Bauer (1972) and Hollander and Wolfe (1999).

\subsection{Control}

We could use the existing Lotus Notes database as control or golden standard, but it could be seen as what Ware (2004) describes as "the somewhat dishonest practice of using a very poor alternative method as a control", since this existing information service (the user interface as well as the database) is outdated in terms of software updates, functionality and possibly hardware as well.

Therefore, after the test subject sees the first mode of the new interface, it is levitated to being the new control, providing a common ground to compare mode 2 and 3 . This is in no way a poor control, since it is the groundwork for the other modes.

\section{Results}

\subsection{Information User}

Out of 24, 13 test subjects were female and 11 male. Participants had an average age of 
39.1 years, with the youngest participant being 23 years old and the oldest 58 . The subjects were employed in a variety of positions ranging from internships to senior management. Seven of the 24 questioned employees stated that they acquired any kind of professional information processing skills. All of them use general search engines like Google and 23 also use professional search engines like scientific databases, 14 of them only for work. All use computers, one person only for work. 22 use smartphones, again one person only for work. 21 individuals have used the old system before, for 15 of them it is part of their daily work.

\subsection{Information Service Quality}

In summary, subjects mentioned 26 distinct negative and 8 distinct positive aspects during the interviews. The 10 most occurring aspects are shown in Table 1. Comments regarding the user interface aside, many participants reported that they enjoyed using the system. For example, one participant said "I like the animations a lot. Also, this overview is very clear ... I like the system" (Survey participant, 2017) or "Interesting things are now visible for me" (Survey participant, 2017). They also reported to "like the design of the system"(Survey participant, 2017). Some verbalized their reservation: "I have problems getting an overview, because the information is displayed so differently" (Survey participant, 2017) or "I can scan a text list more quickly and miss this functionality" (Survey participant, 2017). Another user reported "now I can find out if somebody previously worked with my method or is even doing the same thing at this moment" (Survey participant, 2017) indicating this was not intuitively possible with previous systems.

Table 1: Frequency distribution of critical incident results

$\begin{array}{lr}\text { mentioned aspect } & \text { times mentioned } \\ \text { not using }+20 \text { button } & 14 \\ \text { back button (of the browser) } & 10 \\ \text { some nodes overlap } & 9 \\ \text { no count of results } & 8 \\ \text { bad overview (at any point during the interview) } & 8 \\ \text { good overview } & 8 \\ \text { modern interface/appeal } & 8 \\ \text { start new search by clicking category button } & 7 \\ \text { show attachments only if wanted } & 6 \\ \text { show more (implicit) connections between nodes } & 5\end{array}$


Participants were asked if they would want to see a system on the web, which would show search result visualization similar to the tested prototype. Response options were provided in form of a Likert-scale from 1 to 7 . A high number of the participants (75\%) would be interested in using a similar system on the web. Some (17\%) remained neutral, while $8 \%$ were not interested at all.

The results of the questions on perceived ease of use, usefulness and fun are shown in Figure 3 depicting a flipped bar chart where each bar represents one question. The gray colored area counts responses with the value 4, the middle of the scale and therefore the neutral answer. The bars are centered on this area. Brownish colored bars on the left indicate responses below 4 and the greenish colored bars responses above 4 as shown in the legend. Corresponding percentages for these areas are shown on the left, middle and right side. The users' impression of their own ability to retrieve the required information (ease of use) is highest for the first mode (92\%), with the third mode in second place $(62 \%)$ and lastly the second mode $(46 \%)$. This trend is also reflected for perceived usefulness and perceived fun. Responses for the first mode were the most positive, followed by the third. Finally, the second mode was the least successful for all three aspects. 

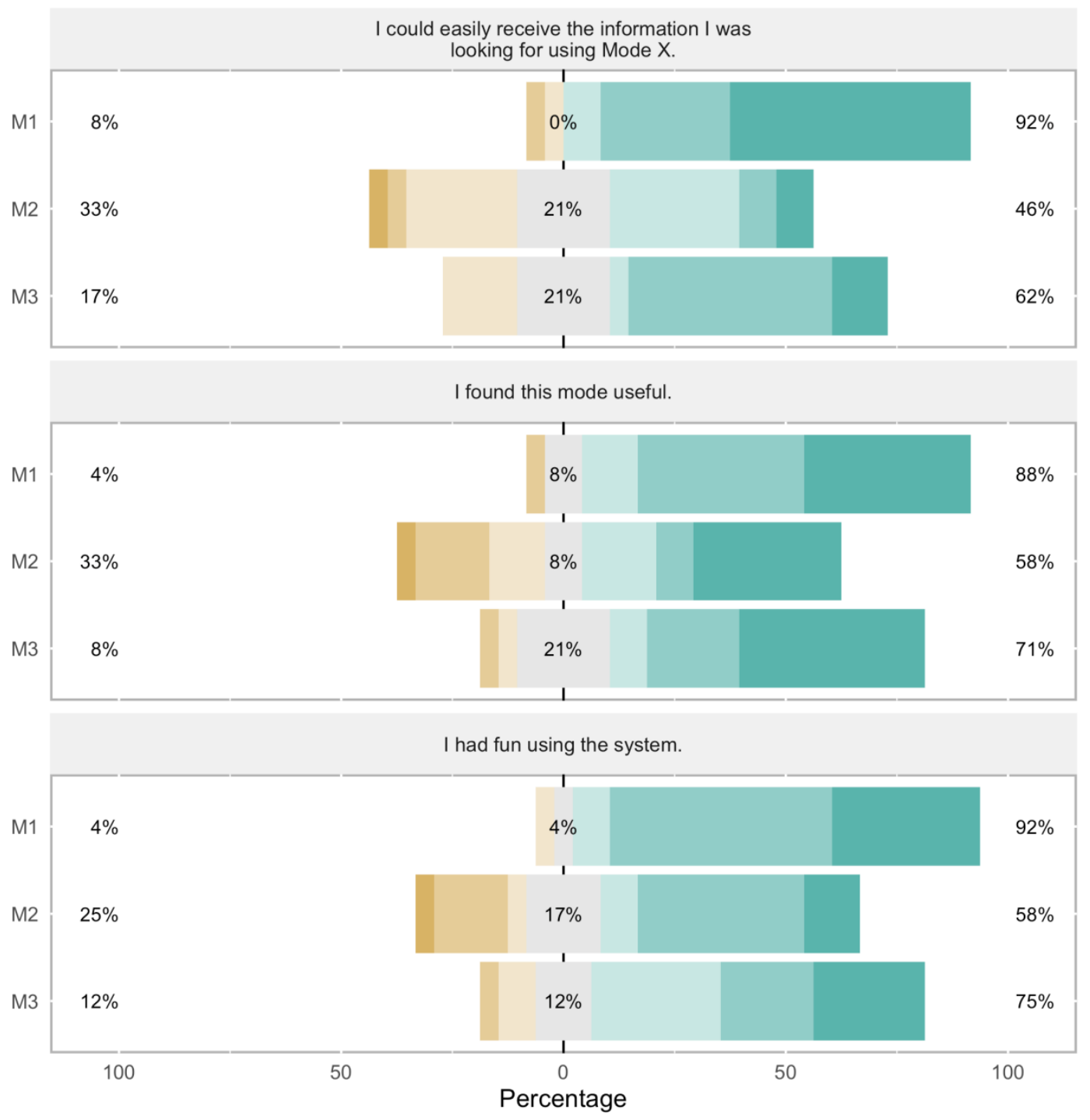

\begin{tabular}{l|l|l|l|l|l|}
\hline Response & 1 & 3 & 5 & 7 \\
& 2 & 4 & 6 &
\end{tabular}

Figure 3: Bar plot of Likert scales for ease of use, usefulness

The statistical analysis shows a significant $(\mathrm{p}<0.05)$ difference in perceived ease of use between mode 1 and the other two modes. For the perceived usefulness and fun, no significant difference could be observed. In summary, users felt they were able to retrieve required information and to do it significantly easier with the first mode (median of 7). They found the modes useful (median of 5, 5 and 6) and had fun using them (median 6, 5.5 and 5). Participants were also asked about their first impression of trustworthiness of the system as a whole, overall response were positive with a median of 5.5 .

Furthermore, test subjects were asked to rank each of the system modes according to their usefulness, including the existing system at the company (Lotus Notes). For consistency, the ranking went from the worst system (1) up to the best system (4). The 
average rating of the existing system (Lotus Notes) was quite low (median of 2) while the third mode got a median of 4 . The Mann-Whitney-Wilcoxon test was applied for comparison of results for the different modes (now including the existing Lotus Notes system). Results show significance values $\mathrm{p}<0.05$ for Lotus Notes compared to mode 3 , mode 1 compared to mode 3 and mode 2 compared to mode 3 . It allows the conclusion of mode 3 being the (significantly) most successful one, especially when compared to the existing text-based system.

We also compare the overall system to the existing Lotus Notes system, meaning any best rated of the three prototyped modes for each test subject against Lotus Notes. Again, the Mann-Whitney-Wilcoxon test is applied. It yields a p-value of .000000084 . The median is 2 for the Lotus Notes system and 4 for the new system. To summarize, there is a significant difference in the ranking of Lotus Notes compared to the new system. For system's performance, on average, Google results took 532ms (with a standard deviation of 202ms), the prototype took $1922 \mathrm{~ms}$ (92ms standard deviation). Additionally, the system did create some errors during the evaluation. This was due to some small delays in query parsing which could be solved by a simple reload of the page. While the system was somewhat slower compared to Google, it still has a low response time. Also, a visual feedback of visual elements being created starts earlier. Test subjects did not complain about slow load times or high error rates.

Next, the results of the task-based testing (usability) are summarized in Table 2. Test subjects were able to answer easy and medium questions very well. As expected, hard questions for the second and third mode were more complex but still approximately half of the test subjects solved them correctly. 


\begin{tabular}{|c|c|c|c|}
\hline & Normal & Q1 & 0 \\
\hline \multirow[t]{5}{*}{ easy } & Normal & Q2 & 1 \\
\hline & Node & Q1 & 0 \\
\hline & Geo & Q1 & 0 \\
\hline & Normal & Q3 & 0 \\
\hline & Node & Q2 & 1 \\
\hline \multirow[t]{3}{*}{ medium } & Geo & Q2 & 2 \\
\hline & Geo & Q3 & 20 \\
\hline & Normal & Q4 & 1 \\
\hline \multirow[t]{2}{*}{ hard } & Node & Q3 & 13 \\
\hline & Geo & Q4 & 1 \\
\hline
\end{tabular}

\subsection{Information Acceptance}

The subjects were asked three questions regarding the possible acceptance of the system. We can see a high system acceptance. 15 out of the 24 test subjects would continue using the system as it is, the rest wants to see some improvements. 22 want the company to implement the system and 17 would definitely recommend it to their colleagues.

\subsection{Information Environment}

The following is concluded from observation and personal communication during the cooperation with the company: The company's culture is one of excellence, creativity and openness but also of competition. Supporting innovative ideas, as for example the development of a novel knowledge system, is one way to stay ahead of competitors, as well as inspiring and motivating employees. Employees of the cooperating department were encouraged to take time out of their work schedules to engage in the planning, development and evaluation of the visual information system (Knowde). Updates on the system were part of regular meetings and also management personnel took the time to review the prototype. Here, the visualization was seen as a means to get an overview of the department's structure and to learn more about "who does what." A better overview over these things may reduce redundancy and facilitate collaboration. 
Knowde was not only recognized as a tool for gaining new insight and decision-making, but as a way to keep up with the times of "business 4.0" and to advertise flexibility, modern functional design and innovative spirit to stakeholders. Still a prototype, it remains to be seen whether Knowde and similar systems can compete with or extend existing and future solutions provided by more conventional vendors like IBM Notes and Microsoft Sharepoint. Decision making on this topic is influenced by many aspects. The overall cost of software roll-outs is taken into account as well as compatibility with the existing infrastructure and personnel.

\section{Discussion}

The questionnaire results showed significant differences between the different variations for ease of use and fun. The simplest mode was also the one who was best received. Overall, users gave high ratings for ease of use, usability and fun of the developed prototype, indicating a satisfactory user experience. This may be also due to the use of colors, movements and novelty of the visual system. Future work could feature psychological analysis as partly suggested by Ware (2004).

The direct ranking of the different modes and the old system showed an overall higher rating for the third mode and significant differences to all other modes. This hints at a discrepancy between ease of use and fun in comparison to the overall impression of the system in the direct ranking. Subjects might rate systems higher because they have greater functionality even though they have a harder time using them.

Three participants, however, actually preferred the old system over the prototype. Of course, since they had been using the old system for years and are used to it, working with a new and completely different system can be a daunting task. But this might not be the only reason. Nielsen (1993) states, that today's users have a firm mental model of how a (web) search should look. "Deviating from this expected design almost always causes usability problems."

The high error rate for task-testing of the third mode, which otherwise was well accepted, can be explained in combination with the qualitative feedback. To correctly answer the question, a user had to understand that relevant results can still be missing when a search query has been processed. Specifically, they had to enter the correct search terms, navigate with both axes and use the load-more button until relevant results would appear. Retrospectively, this question should have been rated as one of hard difficulty and helped to reveal a usability deficit.

We are aware that the evaluation and results presented in this work are not representative for information visualization systems nor for any target group connected 
to the interviews. This is mostly but not only due to the small number of interview participants. A representative study might yield different results. Nevertheless, the work on the system evaluation may raise awareness for some interesting aspects of designing, implementing and evaluation of visual information systems. It is meant as one of the first examples on how the challenge of evaluation could be tackled. Of course, it has to be augmented, extended and updated with time.

For data privacy reasons, neither the system nor the used data set can be made publicly available in their current form. But, because of the adaptability and wide availability, future work includes a public version of Knowde, using one or multiple of the many publicly available (scientific) data sets. This version should introduce improvements as suggested by this evaluation followed by a repeated and improved evaluation.

With the dawn of virtual reality in consumer entertainment, started by the Oculus Rift, $3 \mathrm{D}$ visualization systems and specifically $3 \mathrm{D}$ visual knowledge systems could become, again, an area of interest for research. As the entertainment industry drives technical innovation in display technology and input hardware as well as programming frameworks, this allows researchers to profit from these developments (Donalek et al., 2014). For future work on Knowde, we are highly interested to explore how the feedback-improved version could be adapted to $3 \mathrm{D}$ virtual reality or augmented reality. For this, new research on design principles for VR user interfaces and navigation are needed. Again evaluation of virtual reality interaction is not established as of now.

Much of the user interaction with computer interfaces is derived from their analog counterpart in the physical world, an approach called Skeuomorphism, where "an object or feature $[\ldots]$ imitates the design of a similar artifact in another material or technique" (Page, 2014). While many of these design guidelines and user interaction methods are proven and good practice, a trend towards more minimalistic user interface design principles, also called flat design (Page, 2014) becomes visible (Google Inc., n.d.). Especially, since today's digital natives may not relate to them anymore (e.g., floppy disc as a symbol for saving). Instead, they may be ready to detach themselves from conservative system design and embrace a new generation of knowledge systems. Until then, a hybrid approach may be the solution. There is research on systems which feature a hybrid approach (Clarkson, Desai, \& Foley, 2009; Kraker, Kittel, \& Enkhbayar, 2016; Nguyen \& Zhang, 2006), combining a visual representation with conventional text lists. The Open Knowledge Maps (Kraker et al., 2016) project was introduced during the development of Knowde and aligns closely with our understanding of a modern visual knowledge system. In 2013, (Stock \& Stock, 2013) declared, “[u]p to date, search 
engines on the WWW that used search result visualization (e.g. Kart00, which was discontinued in 2010) have not succeeded on the market." We believe this could change in the near future and see the need for rigorous evaluation with careful consideration for both abstract methodology and specific methods and analyses based on the best practices of science.

\section{REFERENCES}

Alexa Internet Inc. (2017). Alexa Top 500 Global Sites. Retrieved from http://www.alexa.com/topsites

Bauer, D. F. (1972). Constructing Confidence Rank Sets Using Statistics. Journal of the American Statistical Association, 67(339), 687-690. https://doi.org/10.2307/2284469

Bederson, B. B., Shneiderman, B., \& Wattenberg, M. (2002). Ordered and Quantum Treemaps: Making Effective Use of 2D Space to Display Hierarchies. ACM Trans. Graph., 21(4), 833-854. https://doi.org/10.1145/571647.571649

Card, S. K., Mackinlay, J. D., \& Shneiderman, B. (Eds.). (1999). Readings in Information Visualization: Using Vision to Think. San Francisco, CA, USA: Morgan Kaufmann Publishers Inc.

Clarkson, E. C., Desai, K., \& Foley, J. D. (2009). ResultMaps: Visualization for search interfaces. IEEE Transactions on Visualization and Computer Graphics, 15(6), 1057-1064. https://doi.org/10.1109/TVCG.2009.176

Cugini, J., Laskowski, S., \& Sebrechts, M. (2000). Design of 3D Visualization of Search Results: Evolution and Evaluation. 12th Annual International Symposium: Electronic Imaging 2000: Visual Data Exploration and Analysis, 3960, 198-210. https://doi.org/10.1117/12.378896

Donalek, C., Djorgovski, S. G., Cioc, A., Wang, A., Zhang, J., Lawler, E., ... Longo, G. (2014). Immersive and collaborative data visualization using virtual reality platforms. 2014 IEEE International Conference on Big Data (Big Data), 609614. https://doi.org/10.1109/BigData.2014.7004282

Ellis, G., \& Dix, A. (2006). An explorative analysis of user evaluation studies in information visualisation. Proceedings of the 2006 AVI Workshop on BEyond Time and Errors Novel Evaluation Methods for Information Visualization BELIV '06, 1. https://doi.org/10.1145/1168149.1168152

Fekete, J.-D., Wijk, J. J. van, Stasko, J. T., \& North, C. (2008). The Value of Information Visualization. In Information Visualization (pp. 1-18). Springer, Berlin, Heidelberg. https://doi.org/10.1007/978-3-540-70956-5_1

Google Inc. (n.d.). Skeuomorphism, Flat design - Explore - Google Trends. Retrieved 
from https://trends.google.de/trends/explore?q=Skeuomorphism,Flat design Grierson, H. J., Corney, J. R., \& Hatcher, G. D. (2015). Using visual representations for the searching and browsing of large, complex, multimedia data sets. International Journal of Information Management, 35(2), 244-252. https://doi.org/10.1016/j.ijinfomgt.2014.12.003

Heer, J., \& Boyd, D. (2005). Vizster: Visualizing online social networks. In Proceedings - IEEE Symposium on Information Visualization, INFO VIS (pp. 33-40). https://doi.org/10.1109/INFVIS.2005.1532126

Heer, J., Van Ham, F., Carpendale, S., Weaver, C., \& Isenberg, P. (2008). Creation and collaboration: Engaging new audiences for information visualization. In Lecture Notes in Computer Science (including subseries Lecture Notes in Artificial Intelligence and Lecture Notes in Bioinformatics) (Vol. 4950 LNCS, pp. 92-133). https://doi.org/10.1007/978-3-540-70956-5_5

Hollander, M., \& Wolfe, D. A. (1999). Nonparametric statistical methods (Third Edit). https://doi.org/10.1002/9781119196037

Jamieson, S. (2004). Likert scales : how to (ab)use them. Medical Education, 38(12), 1217-1218. https://doi.org/10.1111/j.1365-2929.2004.02012.x

Klouche, K., Ruotsalo, T., Micallef, L., Andolina, S., \& Jacucci, G. (2017). Visual ReRanking for Multi-Aspect Information Retrieval. In Proceedings of the 2017 Conference on Conference Human Information Interaction and Retrieval CHIIR '17 (pp. 57-66). https://doi.org/10.1145/3020165.3020174

Kraker, P., Kittel, C., \& Enkhbayar, A. (2016). Open Knowledge Maps: Creating a Visual Interface to the World's Scientific Knowledge Based on Natural Language Processing. 027.7 Journal for Library Culture, 4(2). https://doi.org/10.12685/027.7-4-2-157

Kuzon, W., Urbanchek, M. G., \& Mccabe, S. J. (1997). Seven deadly sins of statistical analysis. Journal of Oral and Maxillofacial Surgery, 55(8), 897-898. https://doi.org/10.1016/S0278-2391(97)90377-3

Likert, R. (1932). A technique for the measurement of attitudes. (R. S. Woodworth, Ed.). New York NY.

Nguyen, T. N., \& Zhang, J. (2006). A novel visualization model for web search results. IEEE Transactions on Visualization and Computer Graphics, 12(5), 981-988. https://doi.org/10.1109/TVCG.2006.111

Nielsen, J. (1993). Usability Engineering. San Francisco: Morgan Kaufmann Publishers Inc.

Norman, G. (2010). Likert scales, levels of measurement and the "laws" of statistics. Advances in Health Sciences Education, 15(5), 625-632. https://doi.org/10.1007/s10459-010-9222-y 
Page, T. (2014). Skeuomorphism or flat design: future directions in mobile device User Interface (UI) design education. International Journal of Mobile Learning and Organisation, 8(2), 130-142. https://doi.org/10.1504/IJMLO.2014.062350

Rohrer, R. M., \& Swing, E. (1997). Web-Based Information Visualization. IEEE Computer Graphics \& Applications, 17(4), 52-59. https://doi.org/10.1109/38.595269

Schleußinger, M., \& Henkel, M. (2018). Knowde: A Visual Search Interface. LIS Scholarship Archive. https://doi.org/10.17605/OSF.IO/FN5ST

Schumann, L., \& Stock, W. G. (2014). The Information Service Evaluation (ISE) model. Webology, 11(1). Retrieved from http://www.webology.org/2014/v11n1/a115.pdf

Smith, G., Czerwinski, M., Meyers, B., Robbins, D., Robertson, G., \& Tan, D. S. (2006). FacetMap: A scalable search and browse visualization. In IEEE Transactions on Visualization and Computer Graphics (Vol. 12, pp. 797-804). https://doi.org/10.1109/TVCG.2006.142

Stock, W. G., \& Stock, M. (2013). Handbook of Information Science. Berlin: De Gruyter Saur. https://doi.org/10.1515/9783110235005.416

Survey participant. (2017). Personal communication.

Ware, C. (2004). Information Vizualization: Perception for Design (Second Edi). Elsevier.

Xu, S., Jin, T., \& Lau, F. C. M. (2009). A New Visual Search Interface for Web Browsing. In Proceedings of the Second ACM International Conference on Web Search and Data Mining (pp. 152-161). New York, NY, USA: ACM. https://doi.org/10.1145/1498759.1498821

Yi, J. S., ah Kang, Y., Stasko, J. T., \& Jacko, J. A. (2007). Toward a deeper understanding of the role of interaction in information visualization. IEEE Transactions on Visualization and Computer Graphics, 13(6), 1224-1231. https://doi.org/10.1109/TVCG.2007.70515 